\title{
A continuous-stress tetrahedron for finite strain problems
}

\author{
P. Areias ${ }^{a, b, e}$, T. Rabczuk ${ }^{\text {f,* }}$, F. Carapau ${ }^{\text {c,e }}$, J. Carrilho Lopes ${ }^{\text {d,e }}$ \\ a CERIS/Instituto Superior Técnico, University of Lisbon, Portugal \\ ${ }^{\mathrm{b}}$ Department of Physics, Portugal

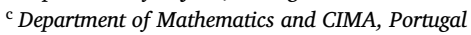 \\ d Department of Geosciences, Portugal \\ e University of Évora Colégio Luís António Verney Rua Romão Ramalho, 59 7002-554, Évora, Portugal \\ ${ }^{\mathrm{f}}$ Institute of Structural Mechanics Bauhaus-University, Weimar Marienstraße, 15 99423, Weimar, Germany
}

\section{A R T I C L E I N F O}

\section{Keywords:}

Hellinger-reissner variational principle

Tetrahedron

Continuous stress

Finite strains

Inf-sup

\begin{abstract}
A B S T R A C T
A finite-strain tetrahedron with continuous stresses is proposed and analyzed. The complete stress tensor is now a nodal tensor degree-of-freedom, in addition to displacement. Specifically, stress conjugate to the relative GreenLagrange strain is used within the framework of the Hellinger-Reissner variational principle. This is an extension of the Dunham and Pister element to arbitrary constitutive laws and finite strain. To avoid the excessive continuity shortcoming, outer faces can have null stress vectors. The resulting formulation is related to the nonlocal approaches popularized as smoothed finite element formulations. In contrast with smoothed formulations, the interpolation and integration domain is retained. Sparsity is also identical to the classical mixed formulations. When compared with variational multiscale methods, there are no parameters. Very high accuracy is obtained for four-node tetrahedra with incompressibility and bending benchmarks being successfully solved. Although the ad-hoc factor is removed and performance is highly competitive, computational cost is high, as each tetrahedron has 36 degrees-of-freedom. Besides the inf-sup test, four benchmark examples are adopted, with exceptional results in bending and compression with finite strains.
\end{abstract}

\section{Introduction}

Numerical analysis [8] and applied mathematics analysis [12] have long agreed on the solutions for locking of low-order elements under quasi-incompressible (and incompressible) conditions. Mixed displacement-pressure formulations passing the inf-sup stability condition are currently well established, as are solutions for circumventing this condition, cf. [26], which developed into the variational multiscale techniques $[1,32,50]$ now in widespread use with virtually all constitutive laws. More traditional formulations, such as the MINI formulation (continuous pressure + bubble-enriched displacement) [6] and its variants [46] are now still adopted [4] in combination with other techniques, see also [31,41].

Accuracy and stability in the quasi-incompressible case are now well understood (see, e.g. Ref. [7]). Another related goal is the high coarsemesh accuracy in the sense of Belytschko and Bachrach [10] in particular when bending is involved. For hexahedra, high coarse-mesh accu- rate elements for finite strains are available $[5,43,49]$ and only incremental developments are being performed to improve specific aspects, for example stability under compression.

High coarse-mesh accurate tetrahedra are difficult to produce as bending modes cannot be directly added with the incompatible mode method. The closest to a bending-enhanced tetrahedron is the rotationbased tetrahedron by Nodargi et al. [35].

An alternative to enrichment consists in increasing the integration domain by creating pseudo-elements based on tetrahedra sharing a node, an edge or a face. This smoothed finite element technology has been extensively explored recently. For node smoothing, stabilization is required [14,19,21,29,38], for edge smoothing [23,37] and face smoothing [33] stabilization is not required. However, in all three smoothing options, Jacobian densification occurs (cf. Fig. 8 of [21]) and the conventional finite element implementation is disrupted. Although intricate to implement, smoothing strategies result in greatly improved bending performance. Incompressibility is however a differ-

\footnotetext{
* Corresponding author.

E-mail address: timon.rabczuk@uni-weimar.de (T. Rabczuk).
} 
Algorithm 1 Relative Lagrangian formulation (Voigt notation adopted) for elasto(visco) plastic materials. Frame $b$, reference configuration $\Omega_{b}$ and equilibrium configuration $\Omega_{a}$.

Input data

Given $\mathbf{e}_{a b}^{b}$ (Voigt form) $\boldsymbol{R}_{00^{\prime}}$ and $\boldsymbol{R}_{0 b}$

Recover from storage $\mathbf{S}_{b 0}^{0}$ and $\mathbf{e}_{b 0}^{0}$

Frame change from 0 to $\mathrm{b}$ and stress/strain updating

Accumulated Green-Lagrange strain in frame $b$

Right stretch tensor for configuration $\Omega_{b}$

Deformation gradient in frames $b-b$

Deformation gradient in frames $b-0$

Update total strain in frame $b$

Stress in frame $b$

Determine stress

and sensitivity

Determine the total strain in frame 0

Determine the second Piola-Kirchhoff stress in frame 0

Store $\mathbf{S}_{a 0}^{0}$ and $\mathbf{e}_{a 0}^{0}$

Return to the element $\mathbf{S}_{a b}^{b}, C_{a b}$ $\mathbf{e}_{b 0}^{b}=\mathcal{T}_{e}\left(\boldsymbol{R}_{0 b}^{T}\right) \mathbf{e}_{b 0}^{0}$

$\boldsymbol{U}_{b 0}^{b}=\sqrt{2 \boldsymbol{e}_{b 0}^{b}+\boldsymbol{I}} J_{b 0}=\operatorname{det} \boldsymbol{U}_{b 0}^{b}$

$\boldsymbol{F}_{b 0}^{b b}=\boldsymbol{R}_{00^{\prime}}^{T} \boldsymbol{R}_{0 b} \boldsymbol{U}_{b 0}^{b}$

$\boldsymbol{F}_{b 0}^{b 0}=\boldsymbol{F}_{b 0}^{b b} \boldsymbol{R}_{0 b}^{T}$

$\mathbf{e}_{a 0}^{b}=\mathbf{e}_{b 0}^{b}+\mathcal{T}_{e}\left[\left(\boldsymbol{F}_{b 0}^{b b}\right)^{T}\right] \mathbf{e}_{a b}^{b}$

$\boldsymbol{S}_{b b}^{b}=\frac{1}{J_{b 0}} \boldsymbol{T}_{s}\left(\boldsymbol{F}_{b 0}^{b 0}\right) \boldsymbol{S}_{b 0}^{0}$

$\mathbf{S}_{a b}^{b}=\mathbf{S}_{b b}^{b}+\Delta \check{S}_{a}\left(\mathrm{e}_{a b}^{b}\right)$

$C_{a b}=\frac{\partial \Delta \check{S}_{a}}{\partial \mathbf{e}_{a b}^{b}}$

$\mathbf{e}_{a 0}^{0}=\mathcal{T}_{e}\left(\boldsymbol{R}_{0 b}\right) \mathbf{e}_{a 0}^{b}$

$\mathbf{S}_{a 0}^{0}=J_{b 0} \mathcal{T}_{s}\left[\left(\boldsymbol{F}_{b 0}^{b 0}\right)^{-1}\right] \mathbf{S}_{a b}^{b}$ ent matter and some of the recent smoothed finite elements include internal bubbles to avoid locking in the incompressible limit, see Refs. $[34,36]$.

We here take a different approach. By using the Hellinger-Reissner variational principle ( $\mathrm{cf}[8,24]$ ), we proposed a stress and displacementbased element for finite strains which can be viewed as an extension of the linear formulation by Dunham and Pister [20] to deal with any constitutive law in finite strains. With that goal, a specific constitutive updating algorithm is used, filling the technical aspects required to extend the small strain formulation to finite strains. Although computational cost is significant, and the stiffness matrix is unsymmetrical, at the node level, system sparsity is the same as with a displacement-based element and implementation is straightforward.

The manuscript is organized as follows: Section 2 presents the constitutive updating algorithm, which is adopted by our tetrahedron formulation, described in Section 3. Section 3 also shows the convergence of the inf-sup parameter for a block mesh. Section 4 presents 4 benchmarks to assess the performance of the new element and finally, in Section 5 draws the conclusions, with a discussion on the advantages and shortcomings of the present element.

\section{Constitutive updating}

To use a common equilibrium formulation for hyperelasticity and rate-based finite-strain elasto-plasticity is a difficult task, especially when using mixed formulations. For specific pressure/displacement [45] or enhanced strain formulations [5,42,43], this can be performed with multiplicative decomposition of the deformation gradient, $\boldsymbol{F}_{e} \boldsymbol{F}_{p}$ [44]. It is intricate to ensure frame-invariance as sharply noted by Glaser and Armero [22]. However, when stress nodal degrees-of- freedom are present, ambiguities would occur due to the required transformations performed at the element level.

Relative strain measures are therefore convenient for the assumed stress formulation employed in this work. Configuration $\Omega_{0}$ is the initial configuration, $\Omega_{b}$ as the reference configuration and $\Omega_{a}$ as the equilibrium configuration. To these three configurations we associate three frames $0, b$ and $a$, respectively. We introduce local frame $0^{\prime}$ corresponding to the local undeformed configuration $\Omega_{0}$. Therefore, for configuration $\Omega_{0} 0$ is the global frame and $0^{\prime}$ is the local frame. A given tensor $\boldsymbol{T}$ is written, in frame $c$ for reference configuration $\Omega_{b}$ and equilibrium configuration $\Omega_{a}$ as $\boldsymbol{T}_{a b}^{c}$. When the frame is obvious or of no consequence, we omit the superscript $c$.

After introducing the relative deformation gradient between configurations $\Omega_{b}$ and $\Omega_{a}$ as $\boldsymbol{F}_{a b}$ we have:

$\boldsymbol{F}_{a b}=\boldsymbol{F}_{a 0} \boldsymbol{F}_{b 0}^{-1}$

The Green-Lagrange strain between configurations is obviously $\boldsymbol{e}_{a b}=$ $\frac{1}{2}\left(\boldsymbol{F}_{a b}^{T} \boldsymbol{F}_{a b}-\boldsymbol{I}\right)$ and therefore we can write:

$\boldsymbol{e}_{a 0}=\boldsymbol{e}_{b 0}+\boldsymbol{F}_{b 0}^{T} \boldsymbol{e}_{a b} \boldsymbol{F}_{b 0}$

If a frame $b$ is identified by the basis vectors $\boldsymbol{g}_{1 b}, \boldsymbol{g}_{2 b}$ and $\boldsymbol{g}_{3 b}$ written in frame 0 by column, we can write the frame matrix for $b$ as $\boldsymbol{R}_{0 b}=$ $\left[g_{1 b}\left|g_{2 b}\right| g_{3 b}\right]$. This frame matrix for the reference configuration is of course relevant for structural elements, but also anisotropic continuum elements where a frame-of-reference is required. An initial frame $\left(0^{\prime}\right)$ is used for structural elements so that in general $\boldsymbol{R}_{00^{\prime}} \neq \boldsymbol{I}$. Of course, although frames are distinct, configurations coincide $\Omega_{0} \equiv \Omega_{0^{\prime}}$. Change of frame of a given second-order tensor $\boldsymbol{T}$ is obtained in general as: $\boldsymbol{T}^{a}=\boldsymbol{R}_{a b} \boldsymbol{T}^{b} \boldsymbol{R}_{a b}^{T}$. Polar decomposition of the deformation gradient $\boldsymbol{F}_{a 0}$ is 
obtained from the rotation and stretch $\boldsymbol{U}_{a 0}$ :

$\boldsymbol{F}_{a 0}=\boldsymbol{R}_{0^{\prime} a} \boldsymbol{U}_{a 0}$

In the particular case of the deformation gradient, which is a two-point tensor, we have the following change of frames transformation, see also [30]:

$\boldsymbol{F}_{a b}^{c d}=\boldsymbol{R}_{c e} \boldsymbol{F}_{a b}^{e f} \boldsymbol{R}_{f d}$

Which allows the writing of $\boldsymbol{F}_{a 0}$ in frames $b-0$ as

$\boldsymbol{F}_{a 0}^{b 0}=\boldsymbol{R}_{b 0} \boldsymbol{F}_{a 0}^{00}$

This formula is relatively standard [47] and supports the classical corotational method, which here is identified as: $\boldsymbol{F}_{b 0}^{b 0}=\boldsymbol{U}_{b 0}^{0}$. Having defined the relative Green-Lagrange strain $\boldsymbol{e}_{a b}$, which is the traditional GreenLagrange strain assuming that the initial configuration is $\Omega_{b}$, we now use the power conjugacy between the Green-Lagrange strain rate $(\dot{e})$ and the second Piola-Kirchhoff stress $(\boldsymbol{S})$ to obtain:

$\int_{\Omega_{a}} \boldsymbol{S}: \dot{\boldsymbol{e}} \mathrm{d} \Omega_{a}=\int_{\Omega_{0}} \boldsymbol{S}_{a 0}: \dot{\boldsymbol{e}}_{a 0} \mathrm{~d} \Omega_{0}=\int_{\Omega_{b}} \boldsymbol{S}_{a b}: \dot{\boldsymbol{e}}_{a b} \mathrm{~d} \Omega_{b}$

from which we obtain the following relation:

$S_{a b}=\frac{1}{J_{b 0}} \boldsymbol{F}_{b 0} \boldsymbol{S}_{a 0} \boldsymbol{F}_{b 0}^{T}$

where $\boldsymbol{J}_{b 0}=\operatorname{det} \boldsymbol{F}_{b 0}$. Generalizing, we have:

$\boldsymbol{S}_{a c}=\frac{1}{J_{c b}} \boldsymbol{F}_{c b} \boldsymbol{S}_{a b} \boldsymbol{F}_{c b}^{T}$

The Cauchy stress, for example, is identified as $\boldsymbol{S}_{a a}$ and follows the traditional formula:

$\boldsymbol{S}_{a a}=\frac{1}{J_{a b}} \boldsymbol{F}_{a b} \boldsymbol{S}_{a b} \boldsymbol{F}_{a b}^{T}$

We therefore have two choices concerning the constitutive updating at finite strains within this framework:

- Hyperelasticity with frame $b: S_{a 0}^{b}\left(e_{a 0}^{b}\right)$

- Hypoelasticity/inelastic materials with frame $b: S_{a b}^{b}=S_{b b}^{b}+$ $\Delta \check{\boldsymbol{S}}_{a}\left(\boldsymbol{e}_{a b}^{b}\right)$ where $\Delta \check{\boldsymbol{S}}_{a}$ is the incremental stress.

Algorithm 1 summarizes the steps (here only for hypoelasticity), where use is made of Voigt form for symmetric tensors:

$\operatorname{Voigt}\left[\begin{array}{lll}S_{11} & S_{12} & S_{13} \\ S_{12} & S_{22} & S_{23} \\ S_{13} & S_{23} & S_{33}\end{array}\right]=\left\{\begin{array}{l}S_{11} \\ S_{22} \\ S_{33} \\ S_{12} \\ S_{13} \\ S_{23}\end{array}\right\} \Leftrightarrow$

$\operatorname{Voigt}[S]=\mathbf{S}$

We use upright bold format for symmetric tensors represented in Voigt format and the usual slanted bold format for tensors in conventional format.

Omitting the configuration and frame indices, the term $\mathcal{T}_{s}(\boldsymbol{F})$ is calculated as:

$$
\boldsymbol{\mathcal { T }}_{s}(\boldsymbol{F})=\left[\begin{array}{cccccc}
F_{11}^{2} & F_{21}^{2} & F_{31}^{2} & 2 F_{21} F_{11} & 2 F_{31} F_{11} & 2 F_{31} F_{21} \\
F_{12}^{2} & F_{22}^{2} & F_{32}^{2} & 2 F_{22} F_{12} & 2 F_{32} F_{12} & 2 F_{32} F_{22} \\
F_{13}^{2} & F_{23}^{2} & F_{33}^{2} & 2 F_{23} F_{13} & 2 F_{33} F_{13} & 2 F_{33} F_{23} \\
F_{11} F_{12} & F_{21} F_{22} & F_{31} F_{32} & F_{21} F_{12}+F_{11} F_{22} & F_{31} F_{12}+F_{11} F_{32} & F_{31} F_{22}+F_{21} F_{32} \\
F_{11} F_{13} & F_{21} F_{23} & F_{31} F_{33} & F_{21} F_{13}+F_{11} F_{23} & F_{31} F_{13}+F_{11} F_{33} & F_{31} F_{23}+F_{21} F_{33} \\
F_{12} F_{13} & F_{22} F_{23} & F_{32} F_{33} & F_{22} F_{13}+F_{12} F_{23} & F_{32} F_{13}+F_{12} F_{33} & F_{32} F_{23}+F_{22} F_{33}
\end{array}\right]
$$

and $\mathcal{T}_{e}(\boldsymbol{F})=\mathcal{T}_{s}^{T}\left(\boldsymbol{F}^{T}\right)$

\section{Stress-displacement formulation for the tetrahedron}

Specific applications, as well as quasi-incompressible constitutive laws, expose the limitations of low-order displacement-based tetrahedra. For an extensive period of time, traditional low-order mixed elements were a solution for avoiding locking in quasi-incompressible problems. Although quasi-incompressible and incompressible materials are well served by mixed $u-p$ elements satisfying the inf-sup condition (see, the description by Bathe [8]), bending and torsion are not. If only quasi-incompressible materials are of interest, the MINI formulation by Douglas Arnold ([6]), see also Bathe [8] and Cao [15] is a reliable solution with low-order interpolation.

However, to improve on results in bending, we use stress tensor interpolation in a form not distinct from the work of Dunham and Pister [20] in finite strains. Here the two-field Hellinger-Reissner variational principle [24] is adopted. Stress and displacement, as in the original Hellinger-Reissner principle, are the independent fields, with stress being the primary. We therefore introduce the independent stress $\widetilde{\mathbf{S}}_{a b}$ as a nodal degree-of-freedom.

- Stresses are linearly interpolated using the corner nodes.

- Relative strain rates and conjugate stresses are employed.

We start with the complementarity strain energy density $U^{c}\left(\widetilde{\mathbf{S}}_{a b}\right)$ which is used in the Hellinger-Reissner functional (in Voigt form [48]):

$\Pi_{H R}\left(\widetilde{\mathbf{S}}_{a b}, \boldsymbol{u}\right)=-\int_{\Omega_{b}} U^{c}\left(\widetilde{\mathbf{S}}_{a b}\right) \mathrm{d} \Omega_{b}+\int_{\Omega_{b}} \widetilde{\mathbf{S}}_{a b} \cdot \mathbf{e}_{a b}(\boldsymbol{u}) \mathrm{d} \Omega_{b}-W_{\text {ext }}(\boldsymbol{u})$

where dependence on the stress $\widetilde{\mathbf{S}}_{a b}$ and the displacement $\boldsymbol{u}$ is made explicit. In (12), $W_{\text {ext }}(\boldsymbol{u})$ is the work of conservative external forces. Stationarity of (12) with respect to $\widetilde{\mathbf{S}}_{a b}$ and $\boldsymbol{u}$ is made here by using a time-derivative. Hence,

$$
\begin{aligned}
\dot{\Pi}_{H R}\left(\widetilde{\mathbf{S}}_{a b}, \boldsymbol{u}\right)= & \int_{\Omega_{b}}\left[\mathbf{e}_{a b}(\boldsymbol{u})-\widetilde{\mathbf{e}}_{a b}\right] \cdot \dot{\widetilde{\mathbf{S}}}_{a b} \mathrm{~d} \Omega_{b} \\
& +\int_{\Omega_{b}} \widetilde{\mathbf{S}}_{a b} \cdot \dot{\boldsymbol{e}}_{a b}(\boldsymbol{u}) \mathrm{d} \Omega_{b}-\dot{W}_{\text {ext }}(\boldsymbol{u})=0
\end{aligned}
$$

with $\widetilde{\mathbf{e}}_{a b}=\frac{\mathrm{d} U^{c}\left(\widetilde{\mathbf{s}}_{a b}\right)}{\mathrm{d} \widetilde{\mathbf{s}}_{a b}}$. Partitioning the terms dependent on $\dot{\boldsymbol{u}}$ and the terms dependent on $\dot{\widetilde{\mathbf{S}}}_{a b}$, we obtain (omitting the argument $\boldsymbol{u}$ ):

$\int_{\Omega_{b}} \widetilde{\mathbf{S}}_{a b} \cdot \dot{\mathbf{e}}_{a b} \mathrm{~d} \Omega_{b}=\dot{W}_{\text {ext }}$

$\int_{\Omega_{b}}\left[\mathbf{e}_{a b}(\boldsymbol{u})-\widetilde{\mathbf{e}}_{a b}\right] \cdot \dot{\tilde{\mathbf{S}}}_{a b} \mathrm{~d} \Omega_{b}=0$

We now make use of a tangent relation $\dot{\widetilde{\mathbf{S}}}_{a b}=c \cdot \dot{\widetilde{\mathbf{e}}}_{a b}$ where the tangent modulus $C$ is obtained from $U^{c}$ as $C=\left[\frac{\mathrm{d}^{2} U^{c}\left(\widetilde{\mathbf{s}}_{a b}\right)}{\mathrm{d} \widetilde{\mathbf{s}}_{a b}^{2}}\right]^{-1}$. By using

$\left.\begin{array}{c}2 F_{31} F_{21} \\ 2 F_{32} F_{22} \\ 2 F_{33} F_{23} \\ { }_{31} F_{22}+F_{21} F_{32} \\ { }_{31} F_{23}+F_{21} F_{33} \\ { }_{32} F_{23}+F_{22} F_{33}\end{array}\right]$




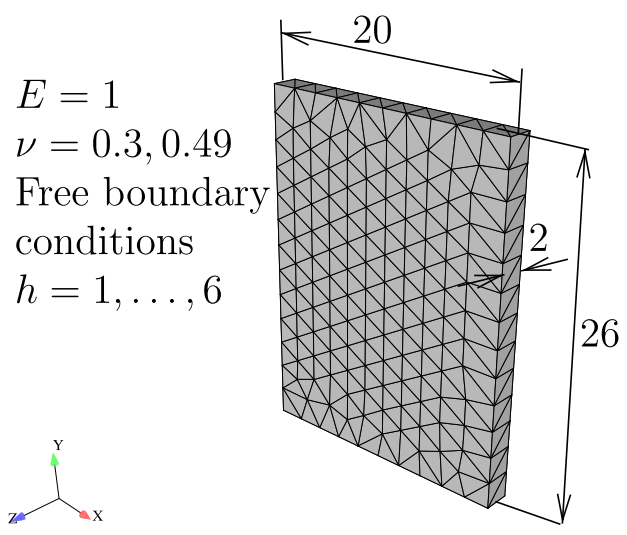

(Consistent units)

(a) Inf-sup test: geometry and properties

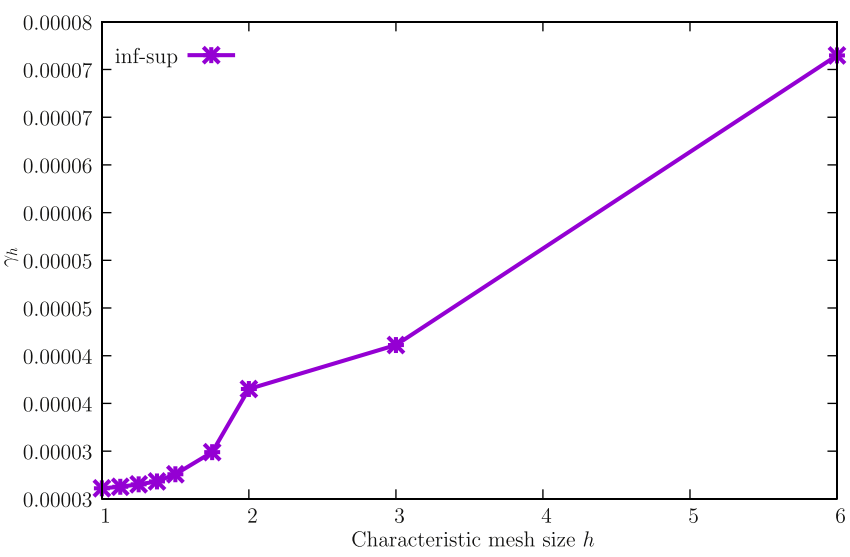

(b) Inf-sup test: convergence of $\gamma_{h}$.

Fig. 1. Inf-sup test $\left(\gamma_{h}\right)$, free boundary conditions. Convergence curve for $\gamma_{h}$.

$\dot{\lambda}=-\dot{\mathbf{e}}_{a b}$ we obtain, by using $\mathbf{e}_{a b}(\boldsymbol{u})-\widetilde{\mathbf{e}}_{a b}=\boldsymbol{O} \Rightarrow \mathbf{S}_{\boldsymbol{a b}}^{b}-\widetilde{\mathbf{S}}_{a b}=\mathbf{0}$,

$\int_{\Omega_{b}}\left[\left(\widetilde{\mathbf{S}}_{a b}-\mathbf{S}_{a b}^{b}\right) \cdot \dot{\lambda}\right] \mathrm{d} \Omega_{b}=0$

In terms of power balance, we use the following relation, where $\widetilde{\mathbf{S}}_{a b}$ is independent of the displacement field. Weak form corresponds to the following stationarity condition:

$\underbrace{\int_{\Omega_{b}} \widetilde{\mathbf{s}}_{a b} \cdot \dot{\mathbf{e}}_{a b} \mathrm{~d} \Omega_{b}+\int_{\Omega_{b}}\left[\left(\widetilde{\mathbf{S}}_{a b}-\mathbf{S}_{a b}^{b}\right) \cdot \dot{\lambda}\right] \mathrm{d} \Omega_{b}}_{\dot{W}_{\text {int }}}=\dot{W}_{\text {ext }}$

where $\boldsymbol{S}_{a b}^{b}$ is the constitutive-based stress and $\lambda$ is now identified as the Lagrange multiplier required to impose, in the weak form, the constraint

$\widetilde{\mathbf{S}}_{a b}$ weak $\cong \mathbf{S}_{a b}^{b}$

In (17), $\dot{W}_{\text {ext }}$ is the external work power, obtained by the internal product of external forces with the corresponding velocities:

$\dot{W}_{\mathrm{ext}}=\int_{\Omega_{b}} \dot{\boldsymbol{u}} \cdot \boldsymbol{b} \mathrm{d} \Omega_{b}+\int_{\Gamma_{t}} \dot{\boldsymbol{u}} \cdot \boldsymbol{t} \mathrm{d} \Gamma_{t}$

where $\dot{\boldsymbol{u}}$ is the velocity vector, $\boldsymbol{b}$ is the external body force and $\boldsymbol{t}$ is the stress vector at boundary $\Gamma_{t}$. Using time-derivatives, in (17), we have $\dot{\lambda}$ as the Lagrange multiplier "velocity" conjugate to the stress.
Table 1

Values of $\gamma_{h}$ for $v=0.3, v=0.499$ and

$h=6,3,1.5,0.75$

\begin{tabular}{lll}
\hline & $v=0.3$ & $v=0.499$ \\
\hline$h=6$ & $7.1478 \times 10^{-5}$ & $7.1478 \times 10^{-5}$ \\
$h=3$ & $4.1122 \times 10^{-5}$ & $4.1122 \times 10^{-5}$ \\
$h=2$ & $3.6516 \times 10^{-5}$ & $3.6516 \times 10^{-5}$ \\
$h=1.75$ & $2.9895 \times 10^{-5}$ & $2.9895 \times 10^{-5}$ \\
$h=1.5$ & $2.7571 \times 10^{-5}$ & $2.7571 \times 10^{-5}$ \\
$h=1.375$ & $2.6827 \times 10^{-5}$ & $2.6827 \times 10^{-5}$ \\
$h=1.25$ & $2.6521 \times 10^{-5}$ & $2.6521 \times 10^{-5}$ \\
$h=1.125$ & $2.6267 \times 10^{-5}$ & $2.6267 \times 10^{-5}$ \\
$h=1$ & $2.6104 \times 10^{-5}$ & $2.6104 \times 10^{-5}$ \\
\hline
\end{tabular}

Using (17), we observed near-singularity in certain problems and therefore modify the equations in weak form to stabilize it while retaining the fully mixed pressure part of the stress:

$\underbrace{\int_{\Omega_{b}} \check{\boldsymbol{S}}_{a b} \cdot \dot{\mathbf{e}}_{a b} \mathrm{~d} \Omega_{b}+\int_{\Omega_{b}}\left[\left(\widetilde{\mathbf{S}}_{a b}-\boldsymbol{S}_{a b}^{b}\right) \cdot \dot{\lambda}\right] \mathrm{d} \Omega_{b}}_{\dot{W}_{\text {int }}}=\dot{W}_{\text {ext }}$

$\check{\boldsymbol{S}}_{a b}=\left[\mathbf{T}_{p}+(1-\varepsilon) \mathbf{T}_{d}\right] \widetilde{\mathbf{S}}_{a b}+\varepsilon \mathbf{T}_{d} \mathbf{S}_{a b}^{b}$

where

$\mathbf{T}_{d}=\frac{1}{3}\left[\begin{array}{cccccc}2 & -1 & -1 & 0 & 0 & 0 \\ -1 & 2 & -1 & 0 & 0 & 0 \\ -1 & -1 & 2 & 0 & 0 & 0 \\ 0 & 0 & 0 & 3 & 0 & 0 \\ 0 & 0 & 0 & 0 & 3 & 0 \\ 0 & 0 & 0 & 0 & 0 & 3\end{array}\right]$

$\mathbf{T}_{p}=\frac{1}{3}\left[\begin{array}{llllll}1 & 1 & 1 & 0 & 0 & 0 \\ 1 & 1 & 1 & 0 & 0 & 0 \\ 1 & 1 & 1 & 0 & 0 & 0 \\ 0 & 0 & 0 & 0 & 0 & 0 \\ 0 & 0 & 0 & 0 & 0 & 0 \\ 0 & 0 & 0 & 0 & 0 & 0\end{array}\right]$

In all examples, we use $\varepsilon=5 \%$. The purpose of this modification is to allow a solution during the Newton iteration, and no effort was made in tuning this parameter. Since the pressure term $\mathbf{T}_{p} \widetilde{\mathbf{S}}_{a b}$ is independent of the interpolated displacement, no locking occurs for any value of $\varepsilon$.

The variation of (20) is required in the application of NewtonRaphson process:

$\mathrm{d} \dot{W}_{\text {int }}=\int_{\Omega_{b}}\left[\mathbf{T}_{p}+(1-\varepsilon) \mathbf{T}_{d}\right] \mathrm{d}_{s} \widetilde{\mathbf{s}}_{a b} \cdot \dot{\mathbf{e}}_{a b} \mathrm{~d} \Omega_{b}$

$+\int_{\Omega_{b}} \varepsilon \mathbf{T}_{d} \mathrm{~d}_{u} \boldsymbol{s}_{a b}^{b} \cdot \dot{\mathbf{e}}_{a b} \mathrm{~d} \Omega_{b}+\int_{\Omega_{b}} \check{S}_{a b} \cdot \mathrm{d}_{u} \dot{\mathbf{e}}_{a b} \mathrm{~d} \Omega_{b}$

$+\int_{\Omega_{b}}\left[\left(\mathrm{~d}_{s} \widetilde{\mathbf{s}}_{a b}-\mathrm{d}_{u} \mathbf{s}_{a b}^{b}\right) \cdot \dot{\lambda}\right] \mathrm{d} \Omega_{b}$

where $d_{S}$ indicates the variation with respect to the stress degrees-offreedom and $\mathrm{d}_{u}$ indicates the variation with respect to the displacement degrees-of-freedom. For a close inspection of the quantities involved in (17) and (23), we have the relative deformation gradient as a product 


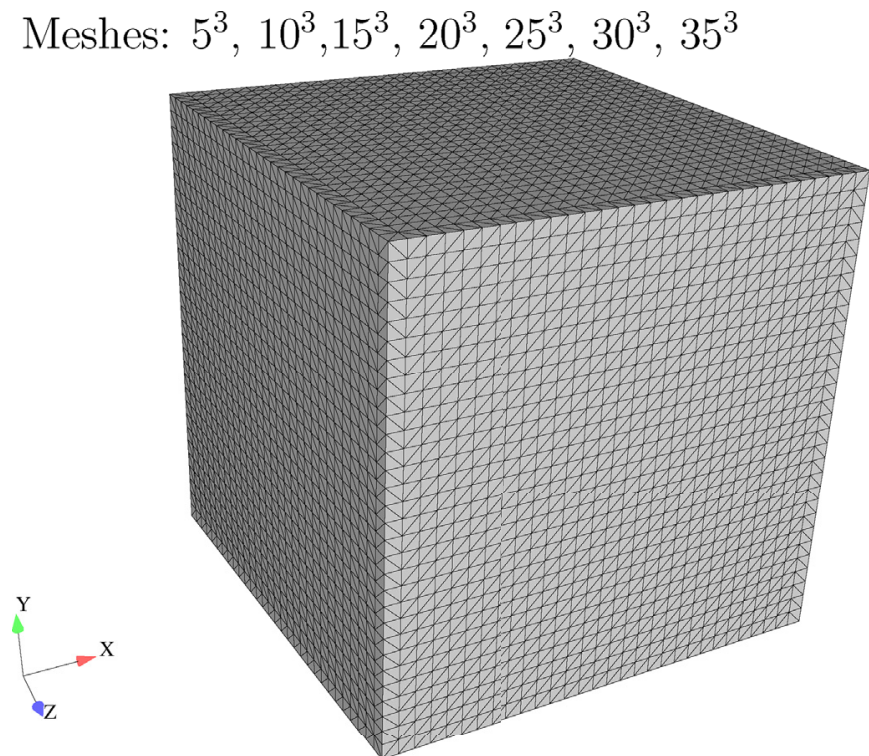

(a) Cube test mesh

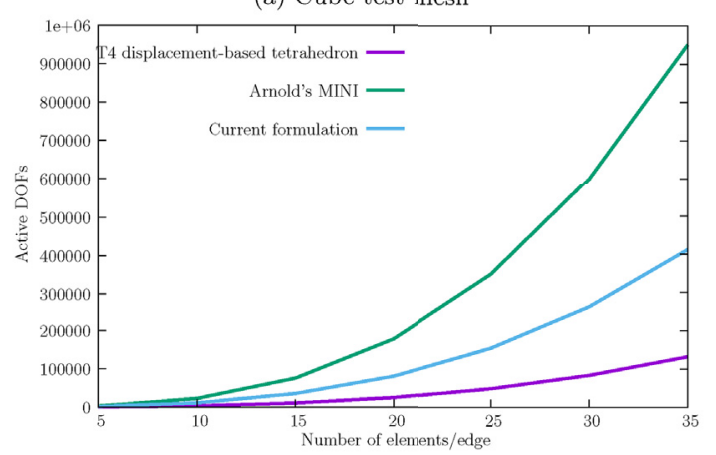

(b) Number of elements per edge vs number of degrees-offreedom.

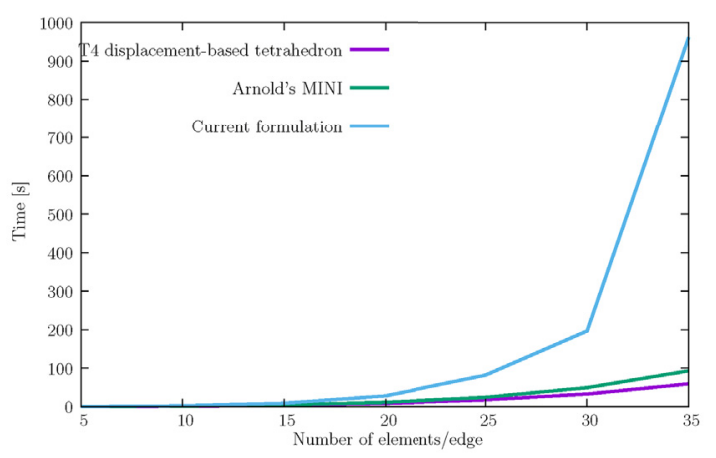

(c) Number of elements per edge vs run time.

Fig. 2. Cube test to assess the computing time.

of the Jacobian for configuration $\Omega_{a}$ with the inverse of the Jacobian for configuration $\Omega_{b}$ :

$\boldsymbol{F}_{a b}=\boldsymbol{J}_{a} \boldsymbol{J}_{b}^{-1}$

Which, introducing the positions at configuration $\Omega_{a}$ and configuration $\Omega_{b}$ as $\boldsymbol{x}_{a}$ and $\boldsymbol{x}_{b}$, along with a set of curvilinear coordinates $\boldsymbol{\xi}$, we obtain:

$J_{a}=\frac{\partial x_{a}}{\partial \xi}$

$J_{b}=\frac{\partial x_{b}}{\partial \xi}$ with the relative displacement being $\boldsymbol{u}_{a b}=\boldsymbol{x}_{a}-\boldsymbol{x}_{b}$. Of course, from (26), we obtain:

$\boldsymbol{e}_{a b}=\frac{1}{2}\left(\boldsymbol{F}_{a b}^{T} \boldsymbol{F}_{a b}-\boldsymbol{I}\right)$

The variation of (29) is given by

$\mathrm{d}_{u} \boldsymbol{e}_{a b}=\frac{1}{2}\left[\boldsymbol{J}_{b}^{-T}\left(\mathrm{~d}_{u} \boldsymbol{J}_{a}^{T} \boldsymbol{J}_{a}+\boldsymbol{J}_{a}^{T} \mathrm{~d}_{u} \boldsymbol{J}_{a}\right) \boldsymbol{J}_{b}^{-1}\right]$

where

$\mathrm{d}_{u} J_{a}=\frac{\partial \mathrm{d}_{u} x_{a}}{\partial \xi}$

In terms of constitutive stress variation, we have:

$\mathrm{d}_{u} \mathbf{s}_{a b}^{b}=C_{a b} \mathrm{~d}_{u} \mathbf{e}_{a b}$

where $\mathcal{C}_{a b}$ is the constitutive tangent modulus. We now introduce the interpolations for $\boldsymbol{u}, \widetilde{\mathbf{s}}_{a b}$ and $\lambda$ as:

$\boldsymbol{u}(\boldsymbol{\xi})=\sum_{K=1}^{4} N_{K}(\boldsymbol{\xi}) \boldsymbol{u}_{K}$

$\widetilde{\mathbf{S}}_{a b}(\boldsymbol{\xi})=\sum_{K=1}^{4} N_{K}(\boldsymbol{\xi}) \widetilde{\mathbf{S}}_{a b K}$

$\lambda(\xi)=\sum_{K=1}^{4} N_{K}(\xi) \lambda_{K}$

We remark that, according to Zienkiewicz, Taylor and Zhu [51], page 296, “... identical interpolation of $\mathrm{N}_{\sigma}$ and $\mathrm{N}_{u}$ is acceptable from the point of view of stability." Shape functions $N_{K}(\xi)$ for each tetrahedron node $K$ are written according to their definition (cf [25]):

$N_{1}(\xi)=1-\xi_{1}-\xi_{2}-\xi_{3}$

$N_{2}(\xi)=\xi_{2}$

$N_{3}(\xi)=\xi_{3}$

$N_{4}(\xi)=\xi_{1}$

Not all quantities are determined by hand-derivation, and we use Mathematica [40] with the AceGen (cf [28]) add-on to calculate some derivatives. Making use of the concepts of Sobolev spaces and norms [13], along with the inf-sup results, we assess the convergence of this element for a simple verification test. We require the $L^{2}$ norm of $\widetilde{\mathbf{S}}_{a b}$ and the Sobolev norm $\|\bullet\|_{W^{1,2}\left(\Omega_{b}\right)}$ of $\boldsymbol{u}$ :

$\left\|\widetilde{\mathbf{S}}_{a b}\right\|_{L^{2}\left(\Omega_{b}\right)}=\sqrt{{\underset{e}{A}}_{\left(\left\|\tilde{\mathbf{S}}_{a b}\right\|_{L^{2}\left(\Omega_{b}^{e}\right)}^{2}\right)_{e}}}$

$\|\boldsymbol{u}\|_{W^{1,2}\left(\Omega_{b}\right)}=\sqrt{\mathbb{e}_{e}^{\mathbb{A}}\left(\sum_{|\alpha|=0}^{1}\left\|\partial^{\alpha} \boldsymbol{u}\right\|_{L^{2}\left(\Omega_{b}^{e}\right)}^{2}\right)_{e}}$

where $\partial^{\alpha} \boldsymbol{u}=\frac{\partial^{|\alpha|} \boldsymbol{u}}{\partial x_{1}^{\alpha_{1}} \partial x_{2}^{\alpha_{2}} \partial x_{3}^{\alpha_{3}}}$ with $|\boldsymbol{\alpha}|=\alpha_{1}+\alpha_{2}+\alpha_{3}$. In (37), $\underset{e}{\mathbb{A}}$ is the assembling operation, described by Hughes [25] with $\Omega_{b}^{e}$ being the reference configuration for element $e$. In terms of components, we have

$\left\|\widetilde{\mathbf{S}}_{a b}\right\|_{L^{2}\left(\Omega_{b}^{e}\right)}^{2}=\int_{\Omega_{b}^{e}} \sum_{i, j=1}^{3}\left(\widetilde{S}_{a b}\right)_{i j}^{2} \mathrm{~d} \Omega_{b}$

$\sum_{|\alpha|=0}^{1}\left\|\partial^{\alpha} \boldsymbol{u}\right\|_{L^{2}\left(\Omega_{b}^{e}\right)}^{2}=\int_{\Omega_{b}^{e}}\left\{\sum_{i=1}^{3}\left[[u]_{i}^{2}+\sum_{k=1}^{3}\left(\frac{\partial[u]_{i}}{\partial X_{k}}\right)^{2}\right]\right\} \mathrm{d} \Omega_{b}$

With (37-38) along with (39-40), we have two discrete quadratic forms for the norms: 


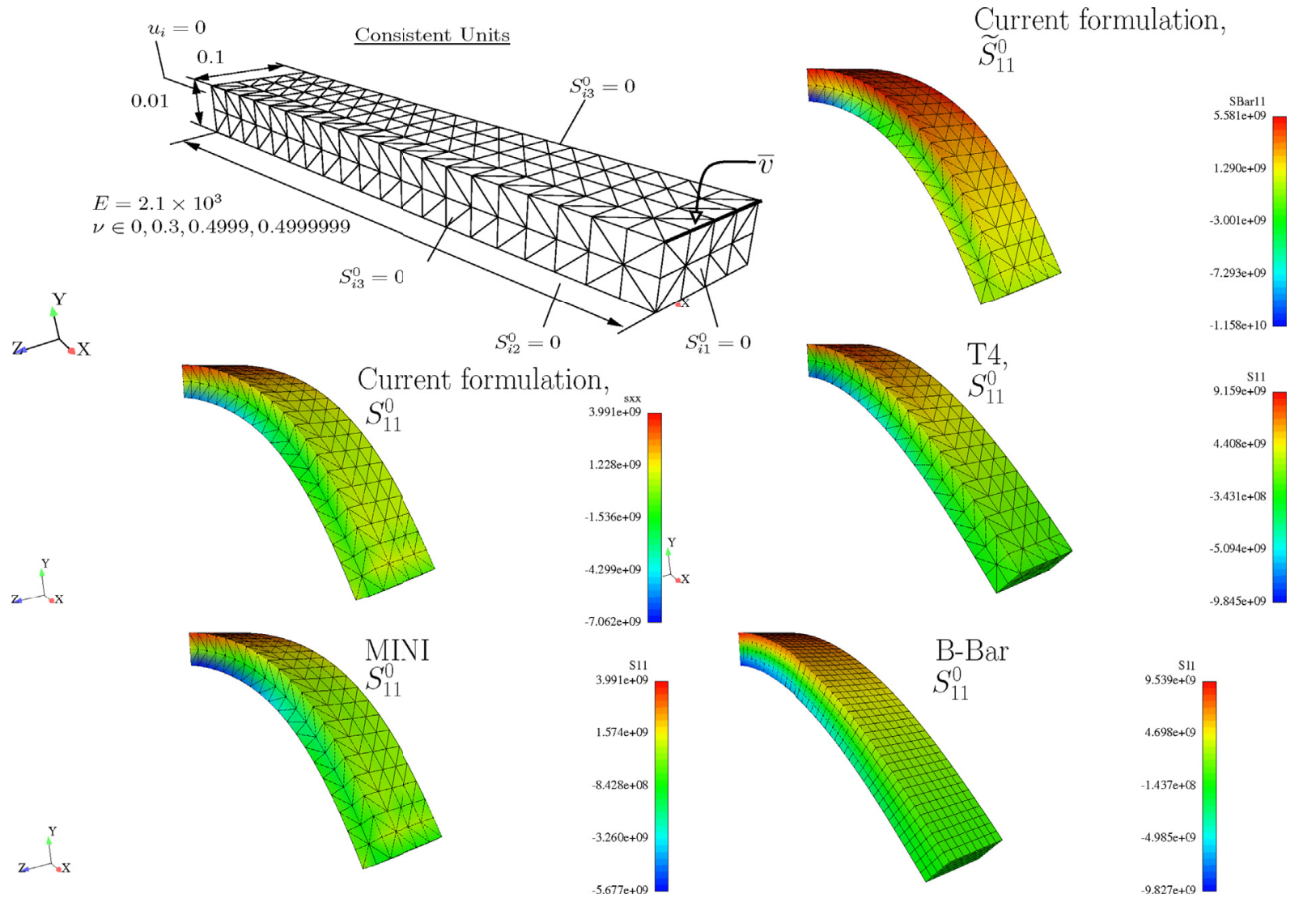

Fig. 3. Cantilever beam: relevant data (geometry, boundary conditions and material properties). Contour plot of $S_{11}^{0}$ stress is also shown for formulations.

$\left\|\widetilde{\mathbf{S}}_{a b}\right\|_{L^{2}\left(\Omega_{b}\right)}^{2}=\widetilde{\mathbf{S}}_{h}^{T} \boldsymbol{G} \widetilde{\mathbf{S}}_{h}$

$\|\boldsymbol{u}\|_{W^{1,2}\left(\Omega_{b}\right)}^{2}=\boldsymbol{u}_{h}^{T} \boldsymbol{T} \boldsymbol{u}_{h}$

where $\boldsymbol{u}_{h}$ and $\widetilde{\mathbf{S}}_{h}$ are the displacement and stress global degrees-offreedom. We note that $\boldsymbol{G}$ and $\boldsymbol{T}$ are global matrices, similar in terms of assembling to the stiffness matrix. We use the assembling operation to perform this task, as well as AceGen [28] to determine the cliques. To that goal, we use the Hessians of $\left\|\widetilde{\mathbf{S}}_{a b}\right\|_{L^{2}\left(\Omega_{b}\right)}^{2}$ and $\|\boldsymbol{u}\|_{W^{1,2}\left(\Omega_{b}\right)}^{2}$ to obtain $\boldsymbol{G}$ and $\boldsymbol{T}$, respectively. Since stress is the primary field, we assess the stability of this formulation in detail, using the inf-sup parameter following Brezzi and Fortin [13] (see Y. Ko and K.-J. Bathe [9,18,27]):

$\gamma_{h}=\operatorname{infsup}_{u_{h}} \widetilde{\mathbf{s}}_{h} \frac{\boldsymbol{u}_{h}^{T} \boldsymbol{K}_{u S} \widetilde{\boldsymbol{s}}_{h}}{\sqrt{\widetilde{\mathbf{s}}_{h}^{T} \boldsymbol{G} \widetilde{\boldsymbol{S}}_{h}} \cdot \sqrt{\boldsymbol{u}_{h}^{T} \boldsymbol{T} \boldsymbol{u}_{h}}}$

This definition provides a number $\gamma_{h}$ that measures the crossed-term in the stiffness matrix and therefore the strength with which the constraint is imposed. We intentionally omit the spaces in (43) to avoid overloading the notation. We now make use of the proof provided by Brezzi and Fortin [11,13] that equates $\gamma_{h}$ to the square-root of the smallest nonzero eigenvalue $\lambda_{k}$ of the following generalized problem, where $\phi_{k}$ are the corresponding eigenvectors:

$\left(\boldsymbol{K}_{u S}^{T} \boldsymbol{T}^{-1} \boldsymbol{K}_{u S}\right) \boldsymbol{\phi}_{k}=\lambda_{k}^{2} \boldsymbol{G} \boldsymbol{\phi}_{k}$

We equate $\gamma_{h}$ to $\min _{\lambda_{k}>0} \lambda_{k}$, see Brezzi and Fortin [13], proposition 3.1. The inf-sup condition is now verified for the geometry shown in Fig. 1. We use a characteristic mesh parameter $h$ with values from 1 to 6 . Table 1 and Fig. $1 \mathrm{~b}$ show the results. Two conclusions are drawn:

- An horizontal asymptote in $\gamma_{h}$ is detected showing the convergence of this formulation.

- The Poisson coefficient does not affect the value of $\gamma_{h}$, a fact that is confirmed in the numerical examples.

In terms of computational cost, we select the most demanding case for sparsity, a cube represented in Fig. 2. A single linear analysis is performed, with upper and bottom faces of the cube being clamped. Meshes with up to 35 elements per edge are adopted. A Clevo Laptop is used, with a 6-core Intel i7-8700 K processor and $32 \mathrm{~GB}$ of $2400 \mathrm{MT} / \mathrm{s}$ RAM. Our linear frontal solver is used, cf [3]. Fig. 2b shows the relation between the number of degrees-of-freedom and the number of elements per edge with elements T4, MINI, and our formulation. We note that in the MINI element, internal degrees-of-freedom are accounted. Clock time vs the number of elements per edge is shown in Fig. 2c. We note that computational cost is significant, which is consequence of using 9 degrees-of-freedom for each node and the stiffness matrix is unsymmetrical.

\section{Numerical tests}

Four numerical experiments are now performed, with a cantilever beam, Cook's membrane, asymmetric compression and the classical drilled plate under tension. The objective is two-fold: verification of the implementation and benchmarking. For comparison, results from the 


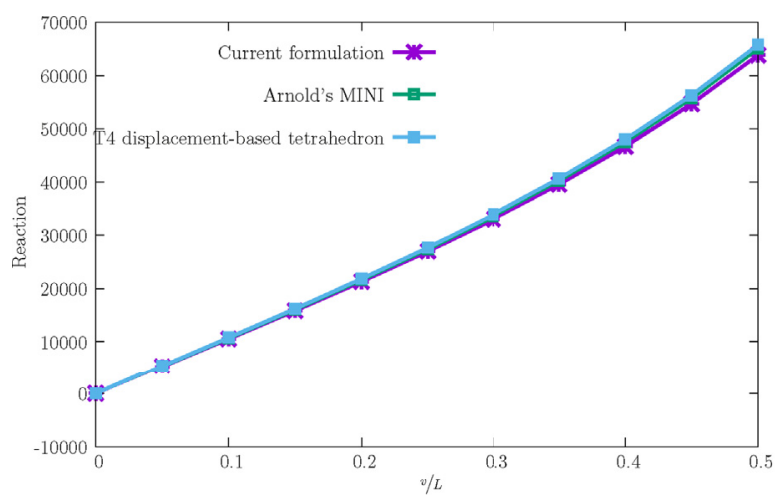

(a) $3 \times 6 \times 30$ elements, $\nu=0.3$

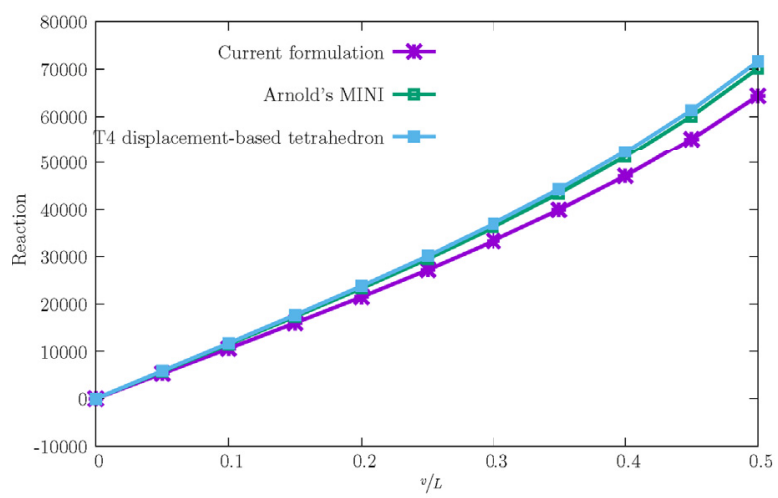

(c) $2 \times 4 \times 20$ elements, $\nu=0.3$

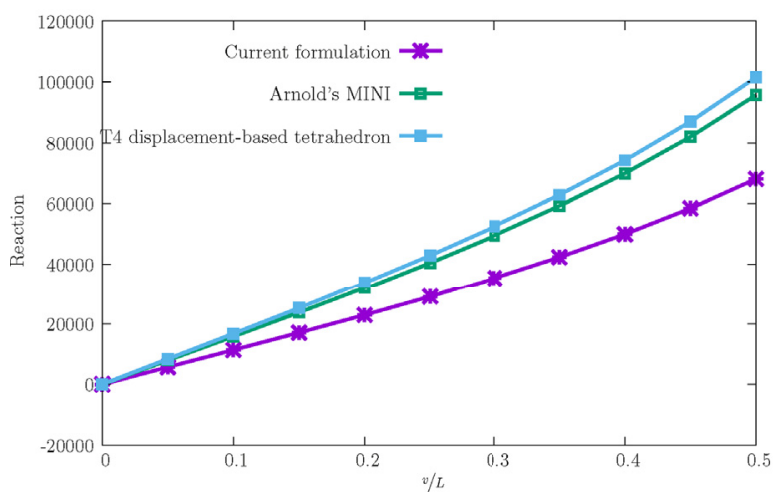

(e) $1 \times 2 \times 10$ elements, $\nu=0.3$

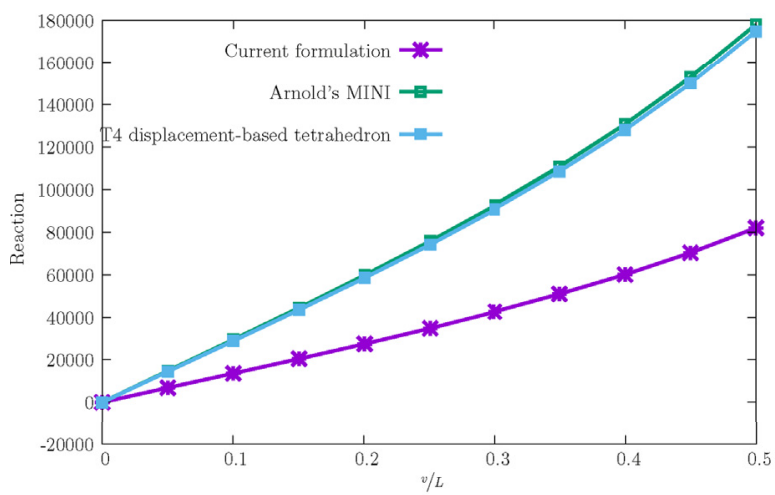

(g) $1 \times 2 \times 5$ elements, $\nu=0.3$

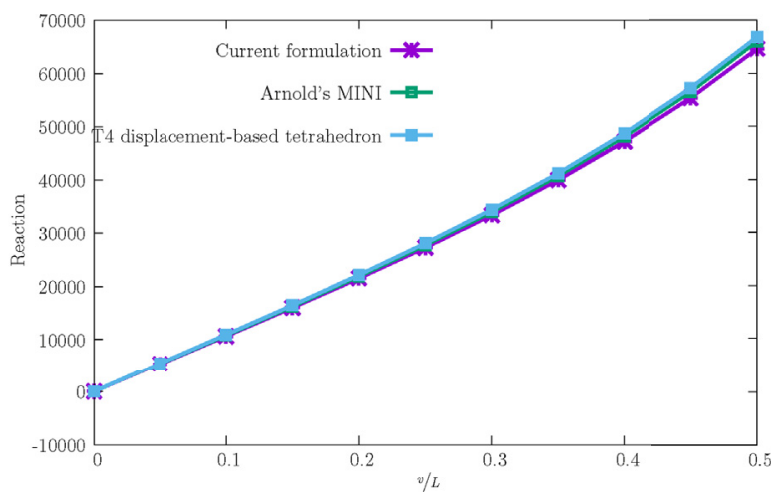

(b) $3 \times 6 \times 30$ elements, $\nu=0.499$

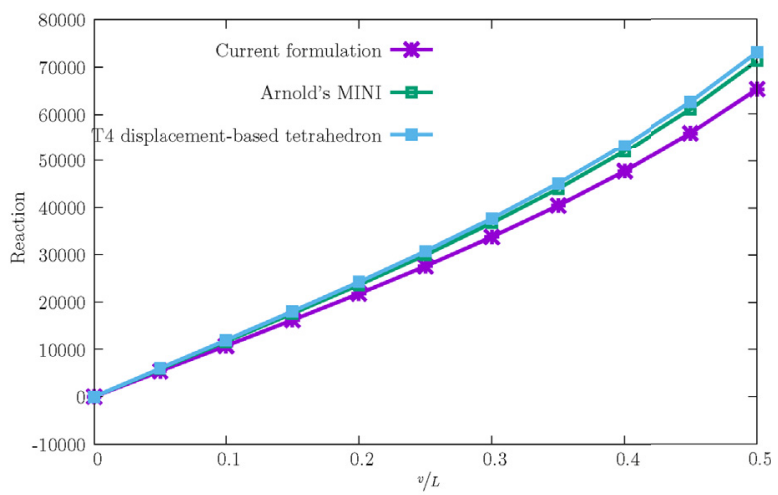

(d) $2 \times 4 \times 20$ elements, $\nu=0.499$

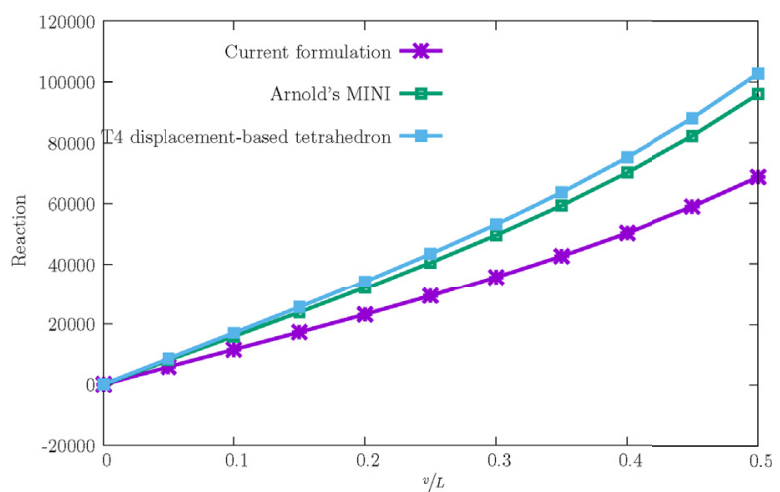

(f) $1 \times 2 \times 10$ elements, $\nu=0.499$

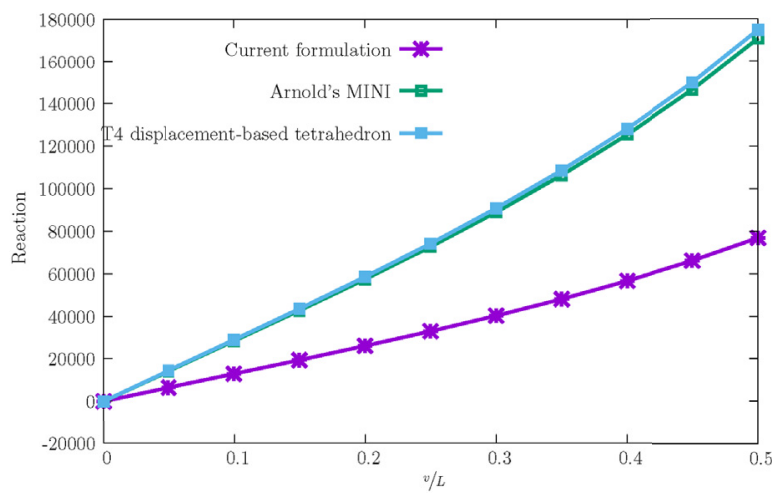

(h) $1 \times 2 \times 5$ elements, $\nu=0.499$

Fig. 4. Cantilever beam: load/displacement results. 

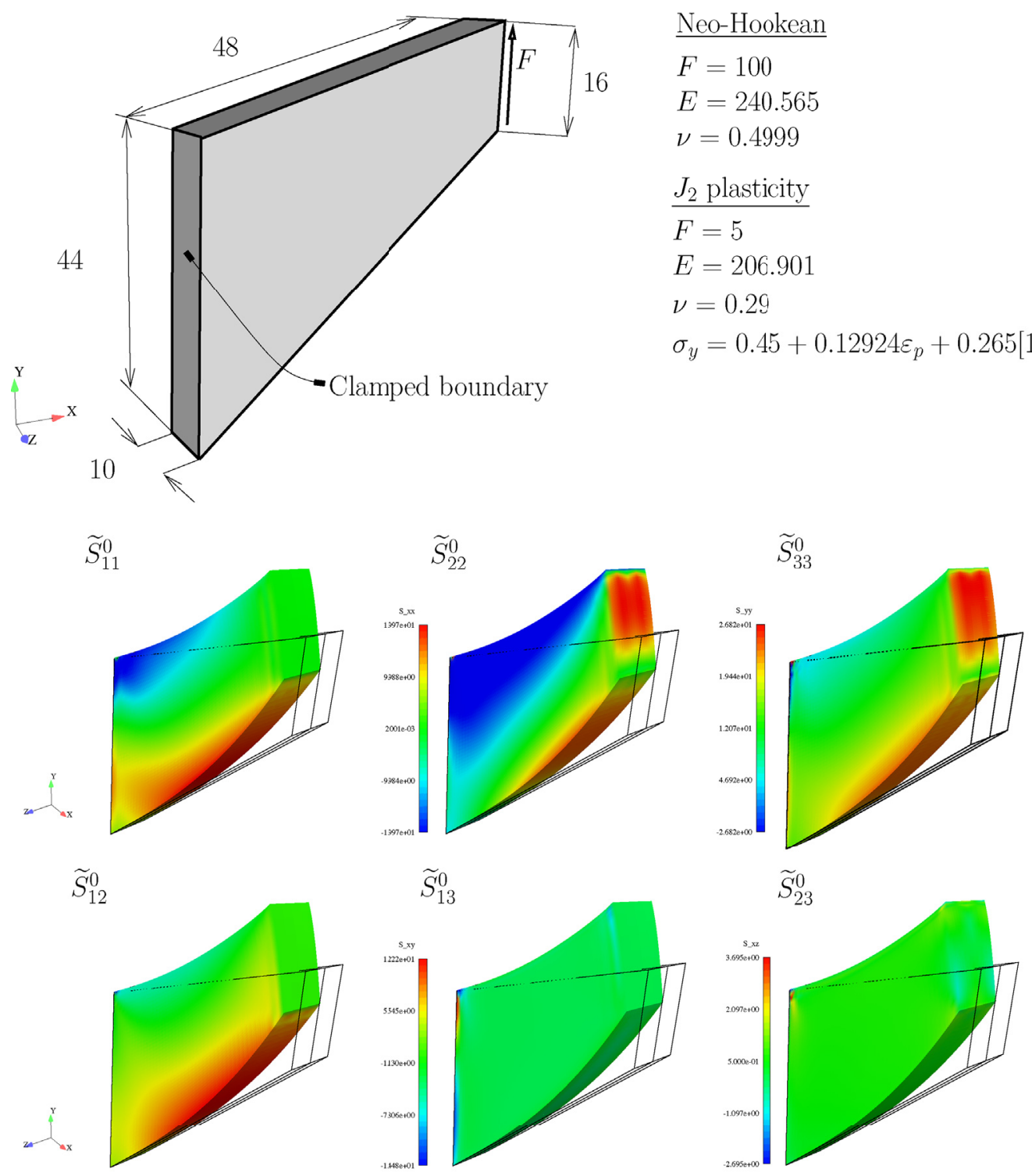

$$
\begin{aligned}
& F=100 \\
& E=240.565 \\
& \nu=0.4999 \\
& \frac{J_{2} \text { plasticity }}{F=5} \\
& E=206.901 \\
& \nu=0.29 \\
& \sigma_{y}=0.45+0.12924 \varepsilon_{p}+0.265\left[1-\exp \left(-16.94 \varepsilon_{p}\right)\right]
\end{aligned}
$$

$\underline{\text { Neo-Hookean }}$
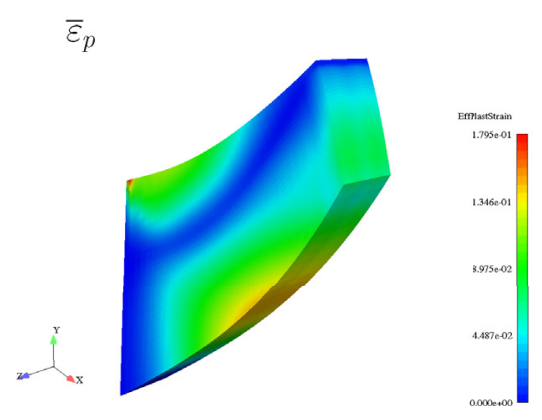

Fig. 5. Cook's membrane: geometry, boundary conditions and constitutive properties.

literature are shown, and critically compared with the ones produced by our formulation. Our SimPlas software (cf [2]) is employed for all examples.

\subsection{Cantilever beam, Kirchhoff/Saint-Venant constitutive law}

Bending tests are especially demanding for tetrahedra element technology when coarse meshes and incompressibility are present. With the goal of assessing the improvements resulting from the current formula- tion, we present a cantilever beam, see Fig. 3 with prescribed vertical displacement at the top edge of the beam extremity. Also shown in Fig. 3 are contour plots of the stress component $\widetilde{S}_{11}^{0}$.

Results for increasingly coarse meshes are shown in Fig. 4. Meshes with $3 \times 6 \times 30,2 \times 4 \times 20,1 \times 2 \times 10$ and $1 \times 2 \times 5$ elements are employed, with two Poisson coefficients: $v=0.3$ and $v=0.499$. We observe that, for coarse meshes, the present formulation clearly outperforms the T4 (textbook implementation of the displacement-based tetrahedron element) and Arnold's MINI element. 


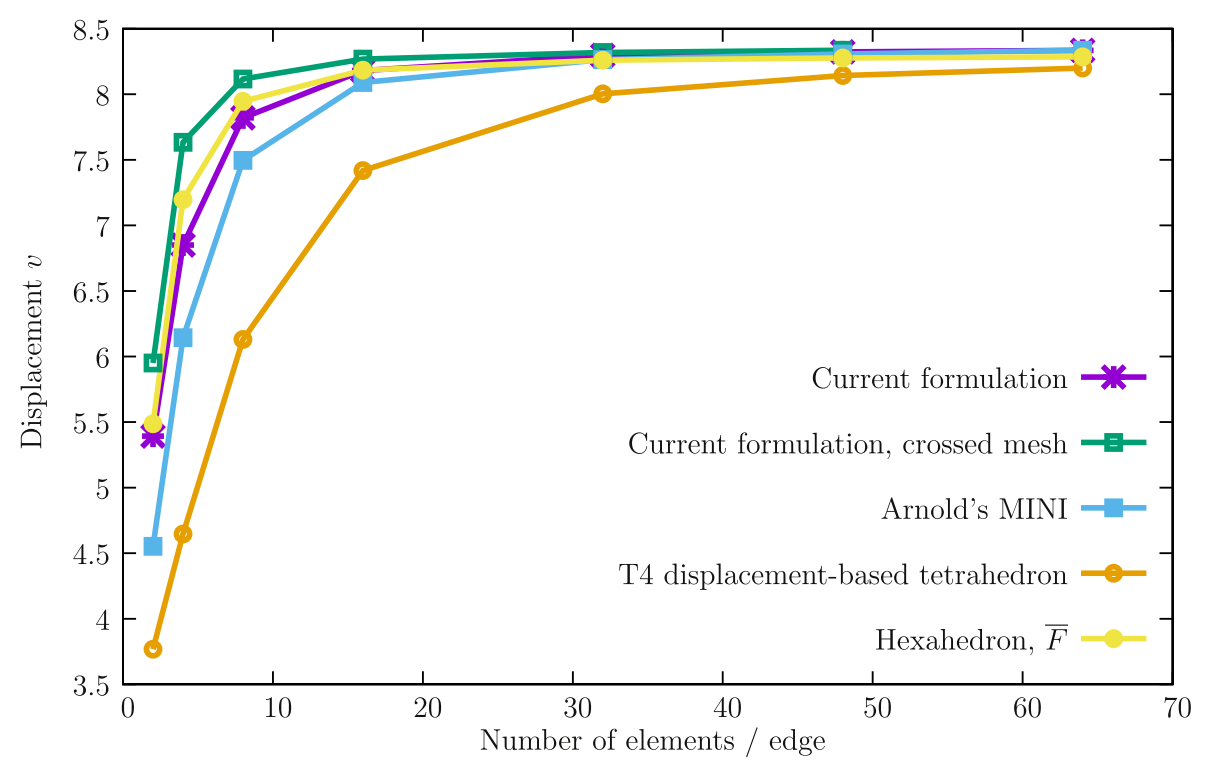

(a) Neo-Hookean

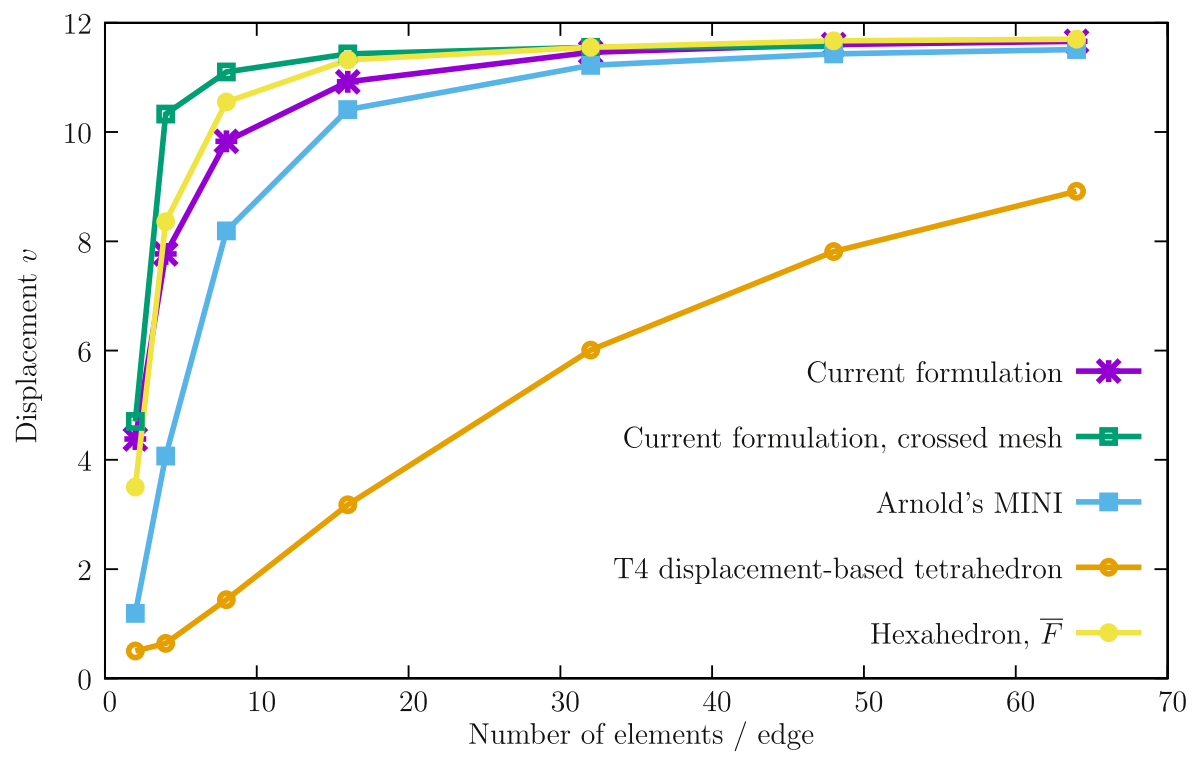

(b) $J_{2}$ elasto-plastic

Fig. 6. Cook's membrane results.

Coarser meshes greatly benefit the present formulation, especially for $v=0.499$.

\subsection{Cook's membrane}

We now apply the formulation to the Cook's membrane problem (the $2 \mathrm{D}$ version is common, ef [32,42]). A 3D linear version was adopted by Mahnken, Caylak, and Laschet [31]. This problem combines incompressibility and bending, which are both especially demanding for loworder tetrahedra. We use two constitutive laws: Neo-Hookean elasticity and $J_{2}$ elasto-plasticity, as described by Simo and Armero [42] to investigate the relative merits when compared with classical formulations. Fig. 5 shows the relevant data and contour plots for the stress degreesof-freedom $\widetilde{S}$ in the elastic and also $\bar{\varepsilon}_{p}$ in the elasto-plastic case. Very smooth stress contour plots are obtained (obviously continuous) which benefits the elasto-plastic analysis. A comparison with the nodally integrated tetrahedra, see Ref. [38], for the same example shows an advantage in terms of stress smoothness. In Fig. 6, we present the results for Neo-Hookean elasticity (sub-Fig. 6a) and $J_{2}$ elasto-plasticity (subFig. 6b). For the new formulation, a standard discretization is used and we also test a crossed-mesh (hexahedra divided into 24 tetrahedra) with the same density. Fig. 6a shows the tip displacement convergence results for the Neo-Hookean case, where a comparison with Arnold's MINI element [6] and the hexahedron version of Simo, Taylor and Pister element [45], here denoted $\bar{F}$, is performed. The new formulation produces high quality results, with the crossed-mesh version outperforming the $\bar{F}$ hexahedron. As expected by the continuity of stresses, the elasto-plastic benchmark produces excellent results, as Fig. $6 \mathrm{~b}$ shows. With the crossed-mesh version, our formulation clearly outperforms the $\bar{F}$ hexahedron in the elasto-plastic test. 
Properties (consistent units)

Quasi-incompressible Neo-Hookean

$\kappa=400889.806$

$\mu=80.194$

Monitored node
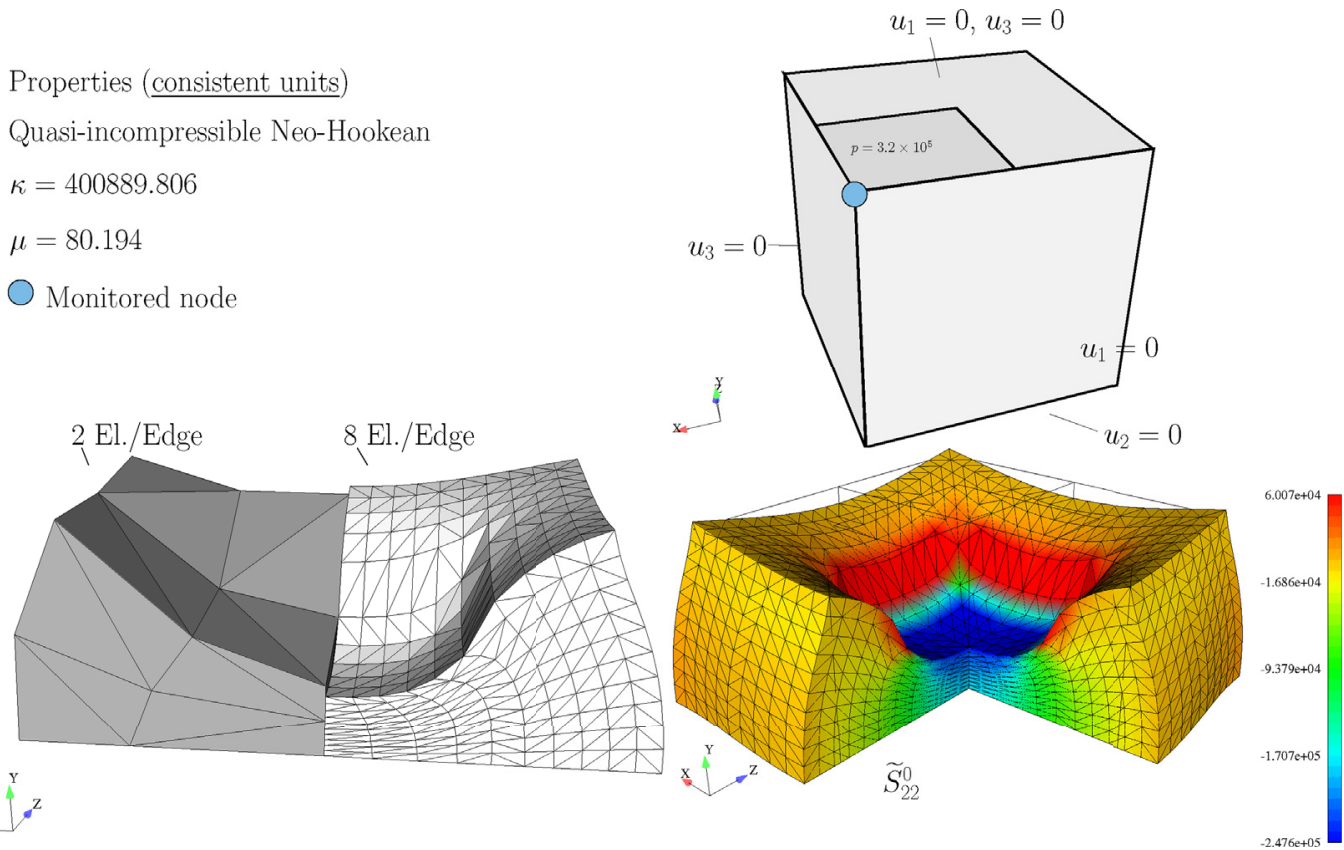

Fig. 7. Quasi-incompressible Neo-Hookean compression test.

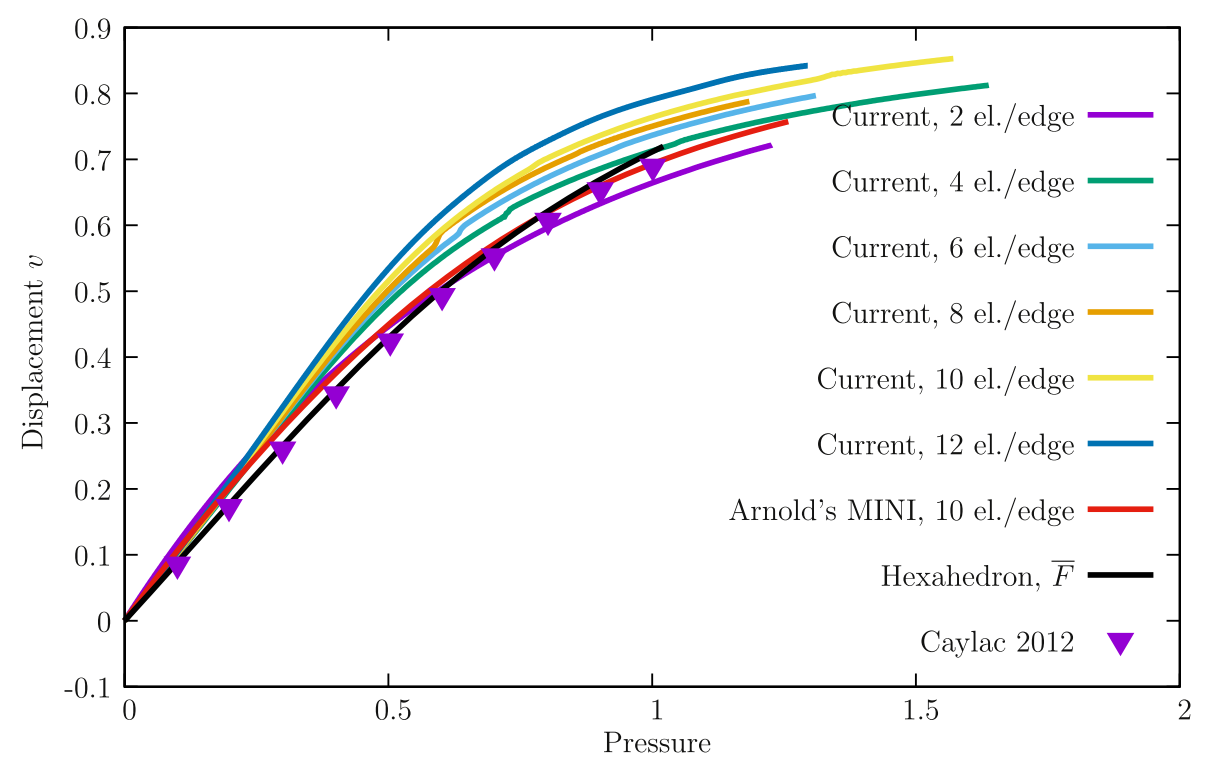

Fig. 8. Quasi-incompressible Neo-Hookean compression test.

\subsection{Quasi-incompressible block under compression}

Using a quasi-incompressible hyperelastic law, we test the new formulation with a well-known benchmark where an asymmetric compression of a block is performed. The large strain compression test by Reese, Wriggers and Reddy [39] and hyper-elastic a quasi-incompressible NeoHookean material law was selected, with the following strain energy density function:

$\Psi=\frac{1}{2} \mu(\operatorname{tr}[\hat{C}]-3)+\frac{1}{2} \kappa(\log [J])^{2}$
In (45), $\widehat{\boldsymbol{C}}=J^{-\frac{2}{3}} \boldsymbol{C}$ and $\boldsymbol{C}=\boldsymbol{F}^{T} \boldsymbol{F}$ with $\kappa=400889.806$ and $\mu=80.194$ (consistent units). In this example, compressive strains and high strain gradients are combined and this poses severe constraints on the element formulation to use. Geometry, constitutive properties and boundary conditions for this example are shown in Fig. 7. One quarter of the system is discretized due to the presence of two symmetry planes. In addition, the upper surface is prescribed in the $x$ and $z$ directions. Relevant properties and dimensions for this problem are shown in Fig. 7. We also show the deformed configurations and the contour plot for the normal stress $\widetilde{S}_{22}^{0}$. The stress is contour plot is extremely smooth, 

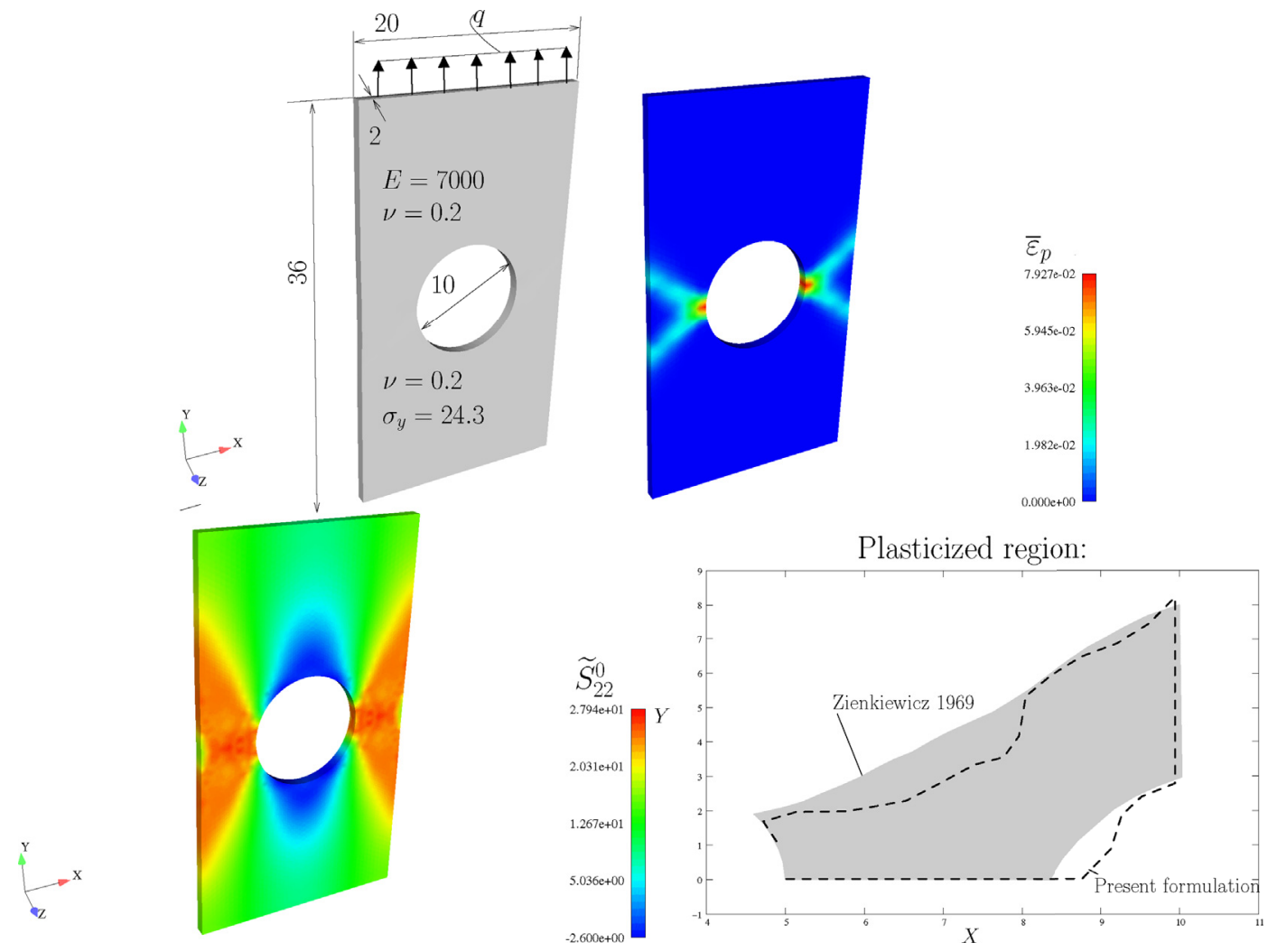

Fig. 9. Drilled plate under tension. Contour plots and loading region comparison.

even in the highly deformed region, confirming the effectiveness of our formulation. Comparing with the results of Masud and Truster [32], our stress contour plots are smoother. Six different meshes are employed, with 2, 4, 6, 8, 10 and 12 elements per edge. Results are shown in Fig. 8 for these meshes and also our implementation of MINI element.

Mesh convergence is excellent, as from 4 elements per edge up all curves coincide. Overall, the results by Caylak and Mahnken [17], using the reduced integration hexahedron Q1R and 8 elements per edge, coincide with our own, as Fig. 8 shows. However, we can use 4 elements per edge and still obtain similar results.

\subsection{Drilled plate under tension}

We now consider an elastic perfectly-plastic benchmark which was employed by Castellazzi, Artioli and Krysl [16] to validate their nodally integrated (element patch-based) NICE-T4 element. This is especially relevant, since their nodally integrated elements entail fewer degreesof-freedom than the present element but produce denser Jacobian matrices. This can be observed in Fig. 2 in Ref. [16]. Krysl's group elements exhibit high performance in demanding problems, such as incompressible and bending-dominated problems. The problem is depicted in Fig. 9 and agrees with the data reported in Ref. [16]. The data also corresponds to the original problem by Zienkiewicz, Vallippan and King [52], who show results for the plastic region in the perfectly-plastic case. Contour plots for $\bar{\varepsilon}_{p}$ and $\widetilde{S}_{22}^{0}$ are shown, as well as a comparison of the plasticized region near the hole are also shown in Fig. 9. There are differences in the domain, when compared with the plot reported by Zienkiewicz, Vallippan and King. Both contour plots are very smooth, a consequence of the use of continuous stress interpolation. In terms of reaction-displacement results, we compare our element and implementation of MINI and T4 elements with that of Castellazzi, Artioli and Krysl. Two meshes are employed (respectively 142 elements and 2998 elements). We can observe in Fig. 10a that the new element performance for the coarse mesh is on-par with a much finer mesh by Castellazzi, Artioli and Krysl. Both MINI and T4 show stiffer results with the coarse mesh. Refining the mesh dilutes the advantage of our present formulation, however it still can be confirmed in Fig. 10b.

\section{Conclusions}

By using the Hellinger-Reissner variational principle (cf [8]), we proposed a stress and displacement-based element for finite strains which can be viewed as an extension of the linear formulation by Dunham and Pister [20] for finite strains. A simple inf-sup numerical test is performed and an horizontal asymptote was obtained, indicating convergence for this formulation.

Compared with alternatives such as the stabilized nodal integration [14,19,21,29,38] and smoothed edges [23,37] and faces [33], no densitification occurs (cf. Fig. 8 of [21]) and the conventional implementation is retained. In contrast with formulations based on bubbles $[4,31,41]$, bending performance is greatly improved. Our formulation follows the traditional finite element assembling process, while excellent results are obtained in bending and incompressibility situations. Under this perspective, it is similar in approach to the nodal rotation technique by Nodargi et al. [35]. Computational cost is significant, but convergence curves show excellent behavior.

Compared with $\bar{F}$ formulations for hexahedra (cf [45]), crossedmesh configurations typically outperform that formulation, even in 


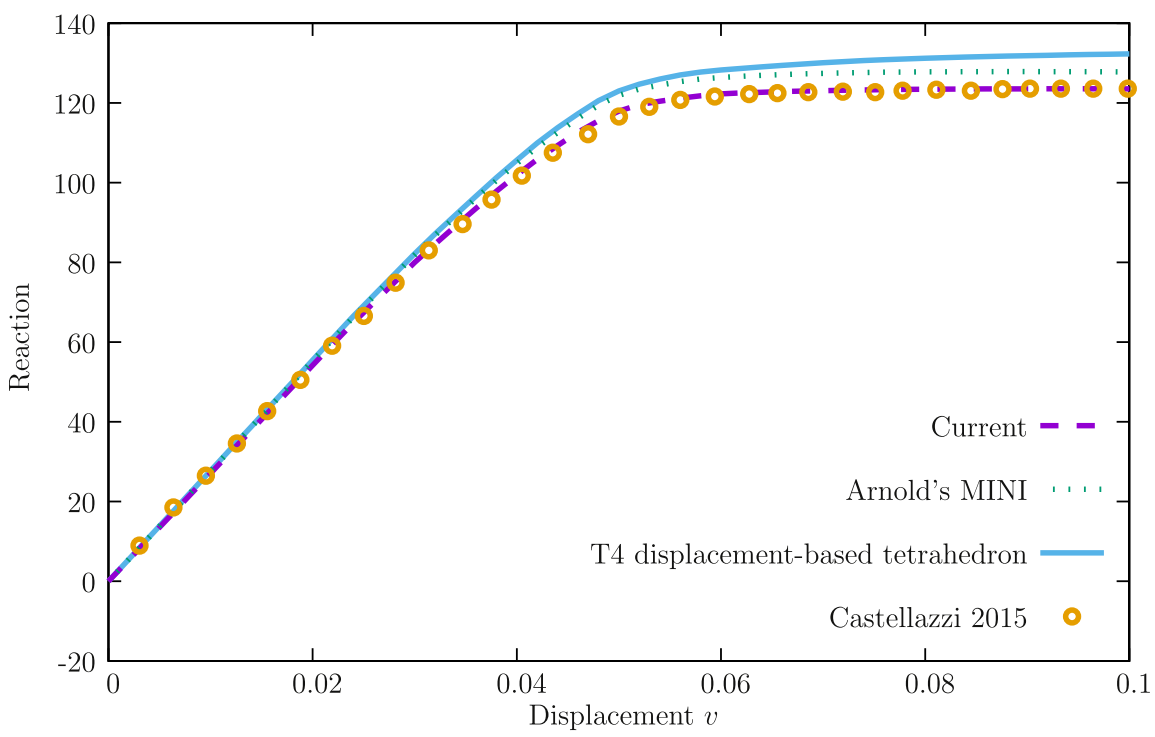

(a) Drilled plate under tension. Coarse mesh (142 nodes, 328 elements).

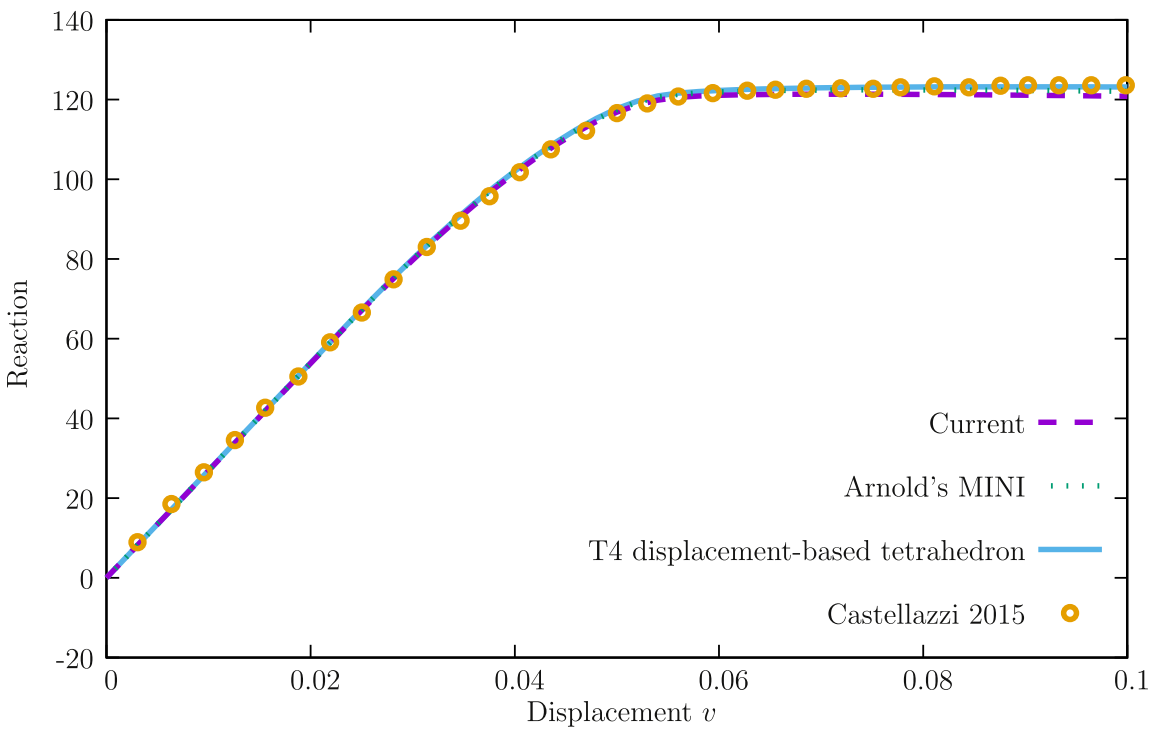

(b) Drilled plate under tension. Fine mesh (2998 nodes, 12630 elements).

Fig. 10. Drilled plate under tension. Comparison with MINI, T4 and NICE-T4 elements for two distinct meshes.

bending-dominated situations. In addition, compared with hexahedra, tetrahedra have the advantage of being suitable for fracture problems and other non-smooth problems.

\section{Appendix A. Supplementary data}

Supplementary data to this article can be found online at https:// doi.org/10.1016/j.finel.2019.07.003.

\section{References}

[1] N. Abboud, G. Scovazzi, Elastoplasticity with linear tetrahedral elements: a variational multiscale method, Int. J. Numer. Methods Eng. 115 (2018) 913-955.

[2] P. Areias. Simplas. http://www.simplas-software.com. Portuguese Software Association (ASSOFT) registry number $2281 / \mathrm{D} / 17$

[3] P. Areias, T. Rabczuk, J.I. Barbosa, The extended unsymmetric frontal solution for multiple-point constraints, Eng. Comput. 31 (7) (2014) 1582-1607.

[4] P. Areias, Matou, Stabilized four-node tetrahedron with, Int. J. Numer. Methods Eng. 76 (2008) 1185-1201.
[5] P.M.A. Areias, J.M.A. Csar de S, C.A. Conceio Antnio, A.A. Fernandes, Analysis of 3D problems using a new enhanced strain hexahedral element, Int. J. Numer. Methods Eng. 58 (2003) 1637-1682.

[6] D.N. Arnold, F. Brezzi, M. Fortin, A stable finite element for the Stokes equations, Calcolo XXI (IV) (1984) 337-344.

[7] F. Auricchio, L. Beiro da Veiga, C. Lovadina, A. Reali, A stability study of some mixed finite elements for large deformation elasticity problems, Comput. Methods Appl. Math. 194 (2005) 1075-1092.

[8] K.-J. Bathe, Finite Element Procedures, Prentice-Hall, 1996.

[9] K.-J. Bathe, The inf-sup condition and its evaluation for mixed finite element methods, Comput. Struct. 79 (2001) 243-252.

[10] T. Belytschko, W.E. Bachrach, Efficient implementation of quadrilaterals with high coarse mesh accuracy, Comput. Methods Appl. Math. 54 (3) (1986) 279-301.

[11] F. Brezzi, K.-J. Bathe, A discourse on the stability conditions for mixed finite element formulations, Comput. Methods Appl. Mech. Eng. 82 (1990) 27-57.

[12] F. Brezzi, M. Fortin, Mixed and Hybrid Finite Element Methods. Number 15 in Springer Series in Computational Mathematics, Springer-Verlag, New York, 1991.

[13] F. Brezzi, M. Fortin, Mixed and Hybrid Finite Element Methods. Number 15 in Springer Series in Computational Mathematics, Springer, New York, 1991.

[14] M. Broccardo, M. Micheloni, P. Krysl, Assumed-deformation gradient finite elements with nodal integration for nearly incompressible large deformation analysis, Int. J. Numer. Methods Eng. 78 (2009) 1113-1134. 
[15] T.S. Cao, P. Montmitonnet, P.O. Bouchard, A detailed description of the Gurson-Tvergaard-Needleman model within a mixed velocity pressure finite element formulation, Int. J. Numer. Methods Eng. 96 (9) (2013) 561-583.

[16] G. Castellazi, E. Artioli, P. Krysl, Linear tetrahedral element for problems of plastic deformation, Meccanica 50 (2015) 3069-3086.

[17] I. Caylak, R. Mahnken, Stabilization of mixed tetrahedral elements at large deformations, Int. J. Numer. Methods Eng. 90 (2012) 218-242.

[18] D. Chapelle, K.-J. Bathe, The inf-sup test, Comput. Struct. 47 (1993) 537-545.

[19] C.R. Dohrmann, M.W. Heinstein, J. Jung, S.W. Key, W.R. Witowski, Node-based uniform strain elements for three-node triangular and four-node tetrahedral meshes, Int. J. Numer. Methods Eng. 47 (2000) 1549-1568.

[20] R.S. Dunham, K.S. Pister, A Finite Element Application of the Hellinger-Reissner Variational Theorem. Technical Report AFFDL-TR-68-150, vol. 45433, Air Force Flight Dynamics Laboratory, Wright Patterson AFB, OH, 1968.

[21] M.W. Gee, C.R. Dohrmann, S.W. Key, W.A. Wall, A uniform nodal strain tetrahedron with isochoric stabilization, Int. J. Numer. Methods Eng. 78 (2009) 429-443.

[22] S. Glaser, F. Armero, On the formulation of enhanced strain finite elements in finite deformations, Eng. Comput. 14 (7) (1997) 759-791.

[23] Z.C. He, G.Y. Li, Z.H. Zhong, A.G. Cheng, G.Y. Zhang, G.R. Liu, E. Li, Z. Zhou, An edge-based smoothed tetrahedron finite element method (es-t-fem) for 3d static and dynamic problems, Comput. Mech. 52 (2013) 221-236.

[24] E. Hellinger, Encyclopedia der Mathematishen Wissnschaften, ume 4, Teubner, Leipzig, 1914. chapter Die allgemeine Aussetze der Mechanik der Kontinua.

[25] T.J.R. Hughes, The Finite Element Method. Dover Publications, 2000, Reprint of Prentice-Hall edition, 1987.

[26] T.J.R. Hughes, L.P. Franca, M. Balestra, A new finite element formulation for computational fluid dynamics: V. circumventing the Babuska-Brezzi condition: a stable Petrov-Galerkin formulation of the Stokes problem accommodating equal-order interpolations, Comput. Methods Appl. Math. 59 (8599) (1986).

[27] Y. Ko, K.-J. Bathe, Inf-sup testing of some three-dimensional low-order finite elements for the analysis of solids, Comput. Struct. 209 (2018) 1-13.

[28] J. Korelc, Multi-language and multi-environment generation of nonlinear finite element codes, Eng. Comput. 18 (4) (2002) 312-327.

[29] P. Krysl, B. Zhu, Locking-free continuum displacement finite elements with nodal integration, Int. J. Numer. Methods Eng. 76 (2008) 1020-1043.

[30] I-Shih Liu, On the transformation property of the deformation gradient under a change of frame, J. Elast. 71 (1) (Apr 2003) 73-80.

[31] R. Mahnken, I. Caylak, G. Laschet, Two mixed finite element formulations with area bubble functions for tetrahedral elements, Comput. Methods Appl. Math. 197 (2008) 1147-1165.

[32] A. Masud, T.J. Truster, A framework for residual-based stabilization of incompressible finite elasticty: stabilized formulations and\$overline $\{\backslash$ boldsymbol $\{\mathrm{f}\}\} \$$ methods for linear triangles and tetrahedra, Comput. Methods Appl. Math. 267 (2013) 359-399.

[33] T. Nguyen-Thoi, G.R. Liu, K.Y. Lam, G.Y. Zhang, A face-based smoothed finite element method (FS-FEM) for 3D linear and geometrically non-linear solid mechanics problems using 4-node tetrahedral elements, Int. J. Numer. Methods Eng. 78 (2009) 324-353.

[34] H. Nguyen-Xuan, G.R. Liu, An edge-based smoothed finite element method softened with a bubble function (bES-FEM) for solid mechanics problems, Comput. Struct. 128 (2013) 14-30.
[35] N.A. Nodargi, F. Caselli, E. Artioli, P. Bisegna, A mixed tetrahedral element with nodal rotations for large-displacement analysis of inelastic structures, Int. J. Numer. Methods Eng. 108 (2016) 722-749.

[36] T.H. Ong, C.E. Heaney, C.-K. Lee, G.R. Liu, H. Nguyen-Xuan, On stability, convergence and accuracy of bES-FEM and bFS-FEM for nearly incompressible elasticity, Comput. Methods Appl. Math. 285 (2015) 315-345.

[37] Y. Onishi, R. Lida, K. Amaya, F-bar aided edge-based smoothed finite element method using tetrahedral elements for finite deformation analysis of nearly incompressible solids, Int. J. Numer. Methods Eng. 109 (2017) 1582-1606.

[38] M.A. Puso, J. Solberg, A stabilized nodeally integrated tetrahedral, Int. J. Numer Methods Eng. 67 (2006) 841-867.

[39] S. Reese, P. Wriggers, B.D. Reddy, A new locking-free brick element technique for large deformation problems in elasticity, Comput. Struct. 75 (2000) 291-304.

[40] Wolfram Research Inc, Mathematica, 2007.

[41] I. Romero, M. Bischoff, Incompatible bubbles: a non-conforming finite element solution for linear elasticity, Comput. Methods Appl. Math. 196 (2007) 1662-1672.

[42] J.C. Simo, F. Armero, Geometrically non-linear enhanced strain mixed methods and the method of incompatible modes, Int. J. Numer. Methods Eng. 33 (1992) 1413-1449.

[43] J.C. Simo, F. Armero, R.L. Taylor, Improved versions of assumed strain tri-linear elements for 3D finite deformation problems, Comput. Methods Appl. Math. 110 (1993) 359-386.

[44] J.C. Simo, T.J.R. Hughes, Computational Inelasticity, corrected second printing edition, Springer, 2000.

[45] J.C. Simo, R.L. Taylor, K.S. Pister, Variational and projection methods for the volume constraint in finite deformation elasto-plasticity, Comput. Methods Appl. Math. 51 (1985) 177-208.

[46] R.L. Taylor, A mixed-enhanced formulation for tetrahedral finite elements, Int. J. Numer. Methods Eng. 47 (2000) 205-227.

[47] C. Truesdell, W. Noll, The Non-linear Field Theories of Mechanics, third ed., Springer, 2004.

[48] N. Viebahn, K. Steeger, J. Schrder, A simple and efficient Hellinger-Reissner type mixed finite element for nearly incompressible elasticity, Comput. Methods Appl. Math. 340 (2018) 278-295.

[49] E.L. Wilson, R.L. Taylor, W. P Doherty, J. Ghaboussi, Incompatible displacement models, in: S.J. Fenves, N. Perrone, A.R. Robinson, W.C. Schnobrich (Eds.), Numerical and Computer Models in Structural Mechanics, Academic Press, New York, 1973, pp. 43-57.

[50] X. Zeng, G. Scovazzi, N. Abboud, O. Coloms, S. Rossi, A dynamic variational multiscale method for viscoelasticity using linear tetrahedral elements, Int. J. Numer. Methods Eng. 112 (2016) 19512003.

[51] O.C. Zienkiewicz, R.L. Taylor, J.Z. Zhu, seventh ed., The Finite Element Method. Its Basics \& Fundamentals, ume First, Butterworth-Heinemann, Elsevier, Oxford, OX5 1GB UK, 2013.

[52] O.C. Zienkiewicz, S. Vallippan, I.P. King, Elasto-plastic solutions of engineering problems. initial stress', finite element approach, Int. J. Numer. Methods Eng. 1 (75100) (1969). 